\title{
How much energy can we consume?
}

\author{
Oleg P.Dimitriev ${ }^{*}$ \\ V.Lashkaryov Institute of Semiconductor Physics, Kiev, Ukraine \\ * Corresponding author. Tel: +38044 5259706, Fax: +38044 5255530,E-mail: dimitr@isp.kiev.ua
}

\begin{abstract}
This report considers the global energy consumption from the viewpoint of thermodynamic balance between the Earth and the cosmic environment. To follow such a balance is postulated to be necessary to maintain proper functioning of the biosphere and stable climate conditions on the Earth. This viewpoint implies the following principles of energy consumption: (i) the energy should be consumed from the renewable sources only and (ii) the amount of the consumed energy should not exceed the amount of energy that incomes to the Earth. Three major sources of the renewable energy are considered, i.e., a direct incoming solar irradiation, a chemical energy through products of photosynthesis and, finally, an outgoing radiation from the Earth as a heated body. It is shown that the first and the third sources are potentially the most effective ways of the energy consumption. The last one, however, is not properly developed now. With the first two sources, the energy consumption rate cannot exceed a limit of $10^{17} \mathrm{~W}$, but practically due to technical restrictions it can be set as $10^{14} \mathrm{~W}$, which is approximately an order higher of the contemporary rate of energy consumption by the mankind. Development of the third source of energy is shown to allow us to increase the above limit up to one order of magnitude more. However, one should take great care of using this source of energy since it can affect climate changes also.
\end{abstract}

Keywords: Thermodynamic balance, Renewable energy, Consumption limit

\section{Nomenclature}

$P_{\text {sun }}$ energy power incoming from the sun to the earth-atmosphere system ... $W$

$P_{\text {earth }}$ energy power incoming to/outgoing from the earth surface. .. $W$

$P^{\downarrow}{ }_{\text {org }}$ energy power stored via photosynthesis.. $W$

$P_{s c}$ energy power produced by solar cells.....W
$P_{O 2}$ energy power to produce oxygen through photosynthesis .......................................... W

$\eta_{\text {org }}$ power conversion efficiency of photosynthesis.... $\%$

$\eta_{\text {indir }}$ power conversion efficiency of solar energy used via produ

$\eta_{\text {sc }}$ power conversion efficiency of solar cells $\%$

$N_{A}$ Avogadro's constant. $\mathrm{mol}^{-1}$

\section{Introduction}

The global strategy of energy consumption has not ever been developed or widely accepted yet. However, there are several signals indicating in favor of such a strategy to be assumed in the near future. One signal comes from the fact that the organic fuel supplies (oil and gas) are exhausted and will come to the end in a few tens of years, and even the coal reserves will run out faster than many believe [1]. The second signal comes from the fact that the global climate is now sensitive to the increasing level of energy consumption and carbon dioxide production as a result of such kind of activity, respectively.

This report considers the principles of the global energy consumption from the viewpoint of thermodynamic balance between the Earth and the cosmic environment, which is necessary to maintain stable and unchanged conditions for proper functioning of the biosphere on the Earth. This viewpoint implies the following principles: (i) The energy should be consumed from the renewable sources only; (ii) The amount of the consumed energy should not exceed the natural production of energy through formation of organic fuel, income of solar energy, etc.

Solar energy is considered as a global supplier of the renewable energy on the Earth, which gives rise to formation of all sources of the organic fuel through photosynthesis, as well as 
energy of wind, hydro-energy through local heating of the earth surface, melting of ice, etc. Nuclear energy is not included in this balance since nuclear materials are not renewable and, moreover, nuclear waste still represents a very dangerous factor for the biosphere.

We consider three possible ways of using a solar energy, namely, the use of a direct incoming solar irradiation, the use of an indirect solar energy through products of photosynthesis and, finally, the use of a reemitted energy from the Earth after the heating of the planet and its irradiation as a black body at the heated temperature. It is considered for the first way that although the power of incoming solar irradiation to the Earth is of the order of $10^{17} \mathrm{~W}$, only a small part of that, approximately $10^{14} \mathrm{~W}$, can be utilized by solar cells due to limitations in their power conversion efficiency, as well as limitation in surface area on the Earth suitable for their displacement. The second way of using the energy through products of photosynthesis can also yield a maximum consumption of $10^{14} \mathrm{~W}$ which is equivalent to the energy power production by the biosphere. The total power of human energy consumption is of the order of $10^{13} \mathrm{~W}$ at the moment [2]. With the modern $4 \%$ annual increase of the energy consumption on the Earth it will take about 60 years to reach the maximal possible limit of $10^{14} \mathrm{~W}$. Finally, there is a large domain of the renewable energy resource in the form of the outgoing terrestrial radiation which could yield additional income and shift the above limit of consumption to higher values. The methods to develop this domain will be discussed below. However, such a shift in energy consumption can be accompanied with unpredictable changes in temperature regimes on the Earth surface. Therefore, the energy consumption limit should be postulated anyway as soon as possible in the near future.

\section{Methodology: the energy balance on the Earth}

The law of energy conservation states that any energy can be spent for performing some work and/or heating. The main source of the incoming energy on the Earth is the Sun. The solar radiation reaching the earth surface has an average power per square meter of about $230 \mathrm{~W} / \mathrm{m}^{2}$ and its spectrum extends from the UV to the IR, with the major part of energy lying in the visible range which has the maximum at the wavelength of about $0.5 \mu \mathrm{m}$ (Fig.1). Eventually, an equal amount of energy must be lost from the Earth-atmosphere system if the internal energy of this system remains constant and the climate is stable. Therefore, the Earth emits the terrestrial radiation as a heated body. However, the spectral range of this outgoing radiation is shifted to the IR since the average temperature of the radiative body, i.e., the Earth, is $288^{\circ} \mathrm{K}$ (Fig.1). Although the peak amount of the outgoing terrestrial radiation (which is at the wavelength of $10 \mu \mathrm{m}$ ) is much smaller as compared to the corresponding peak of the incoming solar radiation at $0.5 \mu \mathrm{m}$, namely, $c a$. $7 \times 10^{-3}$ (calculated using the data from ref. [3]) versus $1.5 \times 10^{3} \mathrm{~W} /\left(\mathrm{m}^{2} \mu \mathrm{m}\right)$, respectively, the outgoing radiation has a much wider spectral range, so that the total amounts of the both radiations are equal in accordance with the thermodynamic balance of the planet.

On the other hand, the amount of photons incoming to and outgoing from the Earth is rather different according to the balance of the incoming and outgoing energy, i.e.,

$E(v)=\int h n d v=\left(\sum h v_{i} n_{i}\right)_{s u n}=\left(\sum h v_{k} n_{k}\right)_{e a r t h}$,

where $n_{i}$ is the amount of photons with the frequency $v_{i}, h$ the Planck constant. It can be roughly evaluated this relationship as $\mathrm{N}_{\text {earth }} / \mathrm{N}_{\text {sun }}=20$, where $\mathrm{N}_{\text {earth }}$ is the average number of photons with the wavelength $10 \mu \mathrm{m}$ emitted by the earth-atmosphere system and $\mathrm{N}_{\text {sun }}$ is the average number of photons with the wavelength $0.5 \mu \mathrm{m}$ from the Sun. 


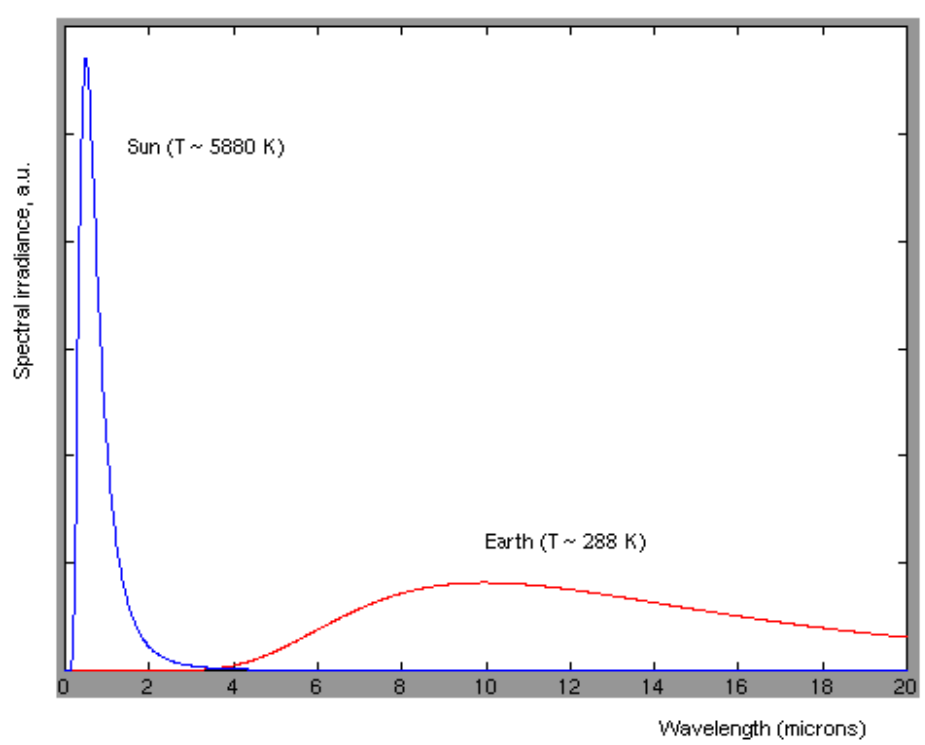

Fig.1. Relationship between incoming solar (blue curve) and outgoing terrestrial (red curve) radiation. The magnitude of the terrestrial radiation is magnified by a factor of 500,000.

However, a small part of photons from the Sun is stored in the form of chemical energy due to photosynthesis. This part of photons can be evaluated taking into account the energy conversion efficiency of photosynthesis to be of the order of $0.1 \%$ (see section 3 for details) that results in only a single stored photon of the thousand ones that are coming from the Sun. As a result, the relationship of the incoming, stored and outgoing energies can be presented schematically in Fig.2. Since only one incoming photon of one thousand gives rise to formation of all organic minerals and fuel, such as coal, natural gas, and petroleum as the products of photosynthesis, it can be easy to understand that only a small part of the incoming energy is mainly consumed now by the mankind.

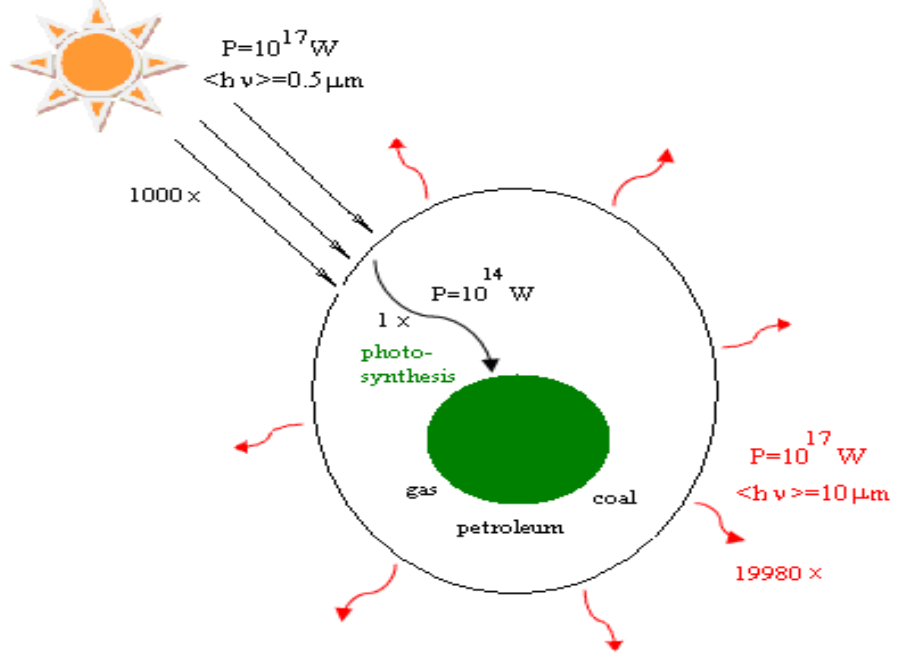

Fig.2. Relationship between incoming, stored, and outgoing energy rates per each 1000 incoming photons from the Sun. 


\section{Use of renewable sources of energy}

\subsection{Indirect consumption of solar energy through products of photosynthesis}

The energy flux utilized by photosynthesis is $\mathrm{P}^{\downarrow}{ }_{\text {org }} \sim 10^{14} \mathrm{~W}$. This value can be calculated from the photosynthesis equation, i.e.

$$
\mathrm{CO}_{2}+\mathrm{H}_{2} \mathrm{O} \Rightarrow \mathrm{CHOH}+\mathrm{O}_{2}
$$

The process described by Eq. (1) involves 4 electrons to be transferred; each of them requires the energy of $1.2 \mathrm{eV}$ per electron. Therefore, formation of an $\mathrm{O}_{2}$ molecule requires consumption of $4.8 \mathrm{eV}$. However, quantum efficiency of the system requires 8 to 12 quanta of light to be adsorbed which results in synthesis of one molecule of oxygen. Therefore, the total energy consumed by the photosynthetic system to produce one molecule of $\mathrm{O}_{2}$ will be approximately $E_{\mathrm{O} 2} \sim 10$ quanta $\mathrm{x} 2 \mathrm{eV}=20 \mathrm{eV}$. Green plants produce totally $10^{14} \mathrm{~kg}$ of $\mathrm{O}_{2}$ per year on the Earth, or $\mathrm{m}_{\mathrm{O} 2}=3 \times 10^{9} \mathrm{~g}$ per second. The energy power necessary to produce $\mathrm{O}_{2}$, therefore, will be

$P_{O 2}=E_{O 2}\left(m_{O 2} / M\right) N_{A}=1.8 \cdot 10^{14} \mathrm{~W}$

where $\mathrm{M}=32$ is the molar mass of $\mathrm{O}_{2}$. Using this result and the power of solar energy reaching the Earth surface which is

$P_{\text {sun }}-$ albedo $(\sim 28 \%)=P_{\text {earth }} \sim 1.2 \cdot 10^{17} \mathrm{~W}$,

one can evaluate the average efficiency of photosynthesis as

$\eta_{\text {org }}=P_{O 2} / P_{\text {earth }} \sim 1.5 \cdot 10^{-3}=0.15 \%$

The same value for $\eta_{\text {org }}$ can be also obtained via expression $P_{\text {org }}^{\downarrow}=\eta_{\text {org }} S_{\text {earth }} \cdot 230 \mathrm{~W} / \mathrm{m}^{2}$, where an assumption is made that the plants cover the overall earth surface $S_{\text {earth }}$. In fact, this assumption is rather crude, because there are large deserts, ice or mountain areas free of plants, but which can be overcompensated, however, by plant diversity in forests which occupy a few levels of space from the earth surface.

The efficiency value (3) can also vary depending on 1 ocal conditions of grow of plant organisms, for example, reaching up to $2 \%$ for water-plants grown in special pools [4].

It is natural to assume that the rate of energy consumption based on products of photosynthesis (gas, oil, coal, etc.), if the concept of energy consumption sets these resources as the basic sources of energy, cannot exceed the rate of formation of the organic products through photosynthesis calculated above. Thus, the energy consumption rate should be limited to $\mathrm{P}_{\text {org }}<10^{14} \mathrm{~W}$ which is only one order of magnitude exceeds the total power of all industrial energy producers currently on the Earth of about $10^{13} \mathrm{~W}$ [5]. The contemporary rate of energy consumption by the mankind can be calculated also by using the data on total mass of burning of dry fuel equivalent to $5 \cdot 10^{12} \mathrm{~kg}$ of carbon per year. The energy of reaction $\mathrm{C}+\mathrm{O}_{2} \Rightarrow \mathrm{CO}_{2}$ is $3.3 \cdot 10^{7} \mathrm{~J} / \mathrm{kg}$ which gives the energy power of $5 \cdot 10^{12} \mathrm{~W}$, which is consistent also with the year energy consumption data of the order of $10^{17} \mathrm{~W} \cdot \mathrm{h}$ [2]. It is easy to calculate, assuming the annual increase of the contemporary energy consumption of about 4\% [5] that the above limit will be reached in the next 60 years $\left((1.04)^{\mathrm{N}}=10\right.$, from where $\left.\mathrm{N}=59\right)$. 
Finally, the efficiency of conversion of the solar energy by indirect way through burning of the organic products of photosynthesis, assuming that power conversion efficiency of the burning process is about $40 \%$, will be only

$$
\eta_{\text {indir }}=6 \cdot 10^{-2} \%
$$

\subsection{Direct consumption of incoming solar radiation}

The power of solar energy irradiated the Earth is of the order of $10^{17} \mathrm{~W}$. That means that the theoretical maximum of the solar energy consumption can be of the same order, i.e., $P_{s c} \sim 10^{17}$ $\mathrm{W}$, if the power conversion efficiency of the corresponding devices approaches $100 \%$. There are limitations, however, because power conversion efficiency of the conventional solar cells is far lower, of the order of $\eta_{s c} \sim 10 \%$, and because these cells cannot be set over the whole surface of the Earth. For example, in order to produce energy comparable with that stored by photosynthesis, the area of the solar cells $\mathrm{S}_{\mathrm{sc}}$ should be as much as $1 \%$ of the Earth surface $\mathrm{S}_{\text {earth}}$, which can be calculated from the following expressions with account of power conversion efficiency for photosynthesis (Eq.(3)),

$P_{s c}=P_{\text {org }}^{\downarrow} \Rightarrow \eta_{s c} S_{s c} \cdot 230 \mathrm{~W} / \mathrm{m}^{2}=\eta_{\text {org }} S_{\text {earth }} \cdot 230 \mathrm{~W} / \mathrm{m}^{2} \Rightarrow S_{s c} / S_{\text {earth }}=\eta_{\text {org }} / \eta_{s c}$

$1 \%$ of the earth surface is rather big, but reasonable value which seems to not significantly affect the biosphere and cropland areas. For comparison, the total urban area on the Earth is now approximately $3.4 \cdot 10^{2} \mathrm{~km}^{2}$ [6] which cover approximately $0.07 \%$ of the Earth surface. To cover an approximately one order of magnitude higher surface seems to be a challenging, but achievable task. However, additional studies are needed to confirm that this value is acceptable and compatible with the living areas on the Earth.

Thus, the energy production through a direct consumption of the solar irradiation energy can reach theoretically one order of magnitude higher value as compared with the contemporary production of energy through burning of organic fuel (see previous section). In addition, power conversion efficiency of the direct conversion of solar energy $\left(\eta_{s c} \sim 10 \%\right)$ is three orders of magnitude higher as compared with that of the indirect production of energy (see Eq.(4)). Thus, a direct consumption of the solar energy is more efficient than the apparent efficiency of burning of the carbon-containing products.

\subsection{Consumption of outgoing terrestrial radiation}

There is a large part of IR energy, $\mathrm{P}^{\uparrow}$ earth, which is reemitted from the earth surface in the form of the terrestrial radiation the Earth emits as the black body with the temperature of $\mathrm{T}_{\text {earth }}=255^{0} \mathrm{~K}$. The amount of this energy is huge and comparable with that coming from the sun to the earth surface, due to the thermodynamic balance of the planet (see Fig.1),

$P_{\text {earth }}^{\uparrow} \sim P_{\text {sun }} \sim 10^{17} \mathrm{~W}$

There are several candidates that can serve as converters of this IR energy to electricity, such as pyroelectric and thermoelectric materials, gapless semiconductors, thermocouples, etc.; however, their application in respect to the terrestrial energy has not been developed yet. The advantages of the consumption of the terrestrial radiation is, first, that this radiation is highly scattered and therefore there is no need for orientation of the corresponding IR receivers or this orientation is not so critical as compared with the solar cells converting direct solar irradiation, so that the IR receivers can occupy several levels upward and, second, they do not 
compete for the solar light with green plants. Below we consider three classes of materials which can be potentially used for the above energy domain.

It is well known that some organic compounds, such as metal complexes, ionic dyes, extended $\pi$-conjugated chromophores, and donor-acceptor charge transfer chromophores can absorbs near-IR (NIR) light with the wavelengths up to $1 \mu \mathrm{m}$ [7]. Recent effort of chemists, however, resulted in development of a new series of donor-acceptor and donor-acceptor-donor D- $\pi$-A$\pi$-D NIR compounds whose absorption can be tuned within the wavelength region of $0.6-1.4$ $\mu \mathrm{m}$ [7]. Even more exciting examples include donor-acceptor covalently linked compounds of tetrathiafulvalene-tetracyanoquinodimethane (TTF- $\sigma$-TCNQ), fused porphyrin ribbons and TTF-dithiolato metal complexes, which demonstrate the absorption spectra extended to the middle-IR with the absorption maxima at $1.6,2.9$, a nd $4.6 \mu \mathrm{m}$, respectively, where the thermo-excited electron transfer has been observed experimentally [8]. Great opportunity can be expected also from the carbon materials and particularly graphene [9] which has a zero band gap and, therefore, can absorb photons from the whole IR range. Thus, design of energy converters based on organic molecules which combine high stability and electronic absorption extended to the middle and far IR is an exciting and challenging problem.

The second class of materials to be used for IR absorption is gapless or narrow-gap semiconductors whose band gap is smaller than $1 \mathrm{e} \mathrm{V}$ (equivalent to $\sim 0.8 \mu \mathrm{m}$ ). Many of semiconductors, such as InSb $(0.17 \mathrm{eV})$, InAs $(0.36 \mathrm{eV}), \mathrm{PbSe}(0.82 \mathrm{eV}), \mathrm{PbTe}(0.31 \mathrm{eV})$ have the band gap values (shown in parenthesis) that allow them to absorb in the near-IR range. However, there are few candidates, i.e., HgSe, HgTe, which can collect energy from the middle IR range also. These last compounds, however, are toxic, and their application, therefore, are to be restricted. Nevertheless, development of these materials in the form of colloidal particles [10] packed in the inert matrix might overcome this problem. The advantages of the nanoparticle application also will give the opportunity to tune the range of absorption by simple change of the particle size.

The above types of the IR receivers produce an electron-hole pair upon absorption of an IR quantum. Their advantage as compared with the solar cells operating in the visible spectrum is that the amount of IR quanta is much larger as compared with the quanta of visible light (see Fig.2); therefore, the IR devices can potentially collect more quanta and produce a 1 arger amount of electron-hole pairs as compared with the conventional solar cells. On the other hand, the devices which can respond in the middle and far IR still should be developed to collect quanta from a more extended spectral range.

The third class of materials perspective for harvesting IR radiation is thermoelectric materials. The thermoelectric energy conversion unit normally consists of two different (n- and p-type) semiconducting materials connected together in the form of a thermocouple; these materials are now actively developed [11], although some skepticism concerning their perspective in the energy solution domain exists [12]. It should be noted that the advantage of thermoelectric devices is that these can consume IR energy of practically all wavelengths and they do not have a quantum threshold restriction from which the device becomes active. However, a sufficiently large thermal gradient is needed for their effective work.

It is a question which part of the IR spectrum is most suitable to consume. If, for example, the corresponding devices will collect IR energy which normally leaves the Earth through the transparency window of the atmosphere (i.e., within the wavelength range of 8-13 $\mu \mathrm{m}$ ) their work can promote the increase in the temperature regime at the earth surface. On the other 
hand, if the IR converters will collect energy in the region of absorption of greenhouse gases and scatter it in the transparency window, their work can weaken the green-house effect and decrease the temperature, respectively. Therefore, the use of the above converters could help in controlling the climate regimes. However, such effects, if any, can be expected only if the converters of IR energy are applied on the large scale.

\section{Conclusions}

The energy consumption rate limit should be set as a necessary concept in the near future to conserve thermodynamic balance on the Earth. Such a limit, if based on the major production of energy through burning of organic fuel, has been shown to exceed the contemporary rate of energy consumption by approximately one order of magnitude and can be reached in the next 60 years. Consumption of direct solar energy and reemitted IR radiation from the Earthatmosphere system on the large scale can somewhat extend the above limit. Optimistic estimates above show that use of the direct solar energy can double this limit. There is no estimate at the moment how much terrestrial radiation can be consumed, since the respective energy converters are at the beginning of their development. It is known, however, that power conversion efficiency of thermoelectric devices is of the order of few percents [12]. The same order of magnitude is typical for the best photovoltaic cells based on organic materials, therefore, it can be expected the same value for power conversion efficiency of the best organic IR converters also. Taking into account some advantages of the IR energy converters discussed above we can suppose very tentatively that their use on the large scale might yield another portion of energy of the order of $10^{14} \mathrm{~W}$. Thus, the overall increase of the total limit of the renewable energy production rate can be very modest, being within $10^{14}-10^{15} \mathrm{~W}$ range. Therefore, the necessary political and economical steps should be undertaken towards the necessity of the energy consumption constraint to keep the living conditions on the Earth constant.

\section{References}

[1] R. Heinberg, D. Fridley, The end of cheap coal, Nature 468, 2010, pp. 367-369.

[2] S.K. Aggarwal, A.K. Gupta, D.G. Lilley, Terrestrial energy, Aerospace America, Dec. 2006, p.70.

[3] C.G. Abbot, Terrestrial temperature and atmospheric absorption, Proc. Natl. Acad. Sci. USA 4, 1918, pp. 104-106.

[4] V.V.Alexeev, K.V.Chekarev, Solar energetics, Nauka, 1991, 60 p.

[5] K.Ya. Kondratyev, I. Galindo, Contemporary stage of civilization development and its perspectives, Univ. of Colima Press, 2002, 139 p.; K. Ya. Kondratyev, I. Galindo, Global change situation: today and tomorrow, Univ. of Colima Press, 2002, 164 p; K.Ya.Kondratyev, Key aspects of global climate change, Energy and Environment 15, 2004, pp. 467-501.

[6] Demographia world urban areas \& population projections: Edition 6.1, July 2010, pp.1128 (www.demographia.com/db-worldua.pdf).

[7] G. Qian, Z. Y. Wang, Near-infrared organic compounds and emerging applications, Chem. Asian J. 5, 2010, pp. 1006-1029.

[8] D. F. Perepichka, M. R. Bryce, Molecules with exceptionally small HOMO-LUMO gaps, Angew. Chem. Int. Ed. 44, 2005, pp. 5370-5373. 
[9] J.M. Dawlaty, S. Shivaraman, J. Strait, P. George, M. Chandrashekhar, F. Rana, M.G. Spencer, D. Veksler, Y. Chen, Measurement of the optical absorption spectra of epitaxial graphene, Appl. Phys. Lett. 93, 2008, 13195.

[10] M. T. Harrison, S. V. Kershaw, M. G. Burt, A. L. Rogach, A. Kornowski, A. Eychmüller, and H. Weller, Colloidal nanocrystals for telecommunications. Complete coverage of the low-loss fiber windows by mercury telluride quantum dots, Pure Appl. Chem. 72, 2000, pp. 295-307.

[11] J.F. Li, W.S. Liu, L.D. Zhao, M.Zhou, High-performance nanostructured thermoelectric materials, NPG Asia Mater. 2, 2010, pp. 152-158.

[12] C.B.Vining, An inconvenient truth about thermoelectrics, Nature Mater., 8, 2009, pp. 8385. 